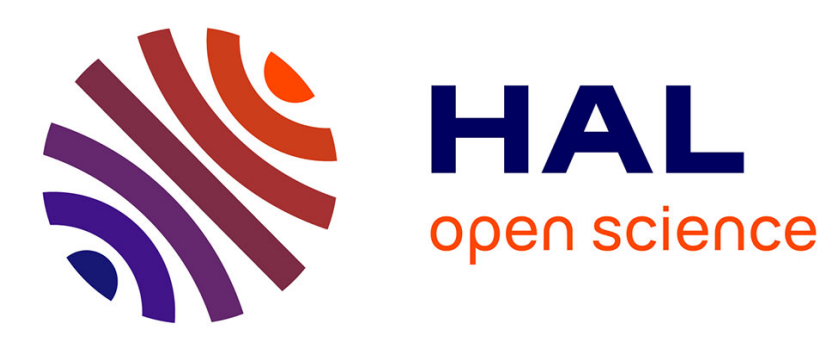

\title{
Stability of the spin density wave phases in (TMTSF)2ClO4 : quantized nesting effect
}

\author{
M. Héritier, G. Montambaux, P. Lederer
}

\section{To cite this version:}

M. Héritier, G. Montambaux, P. Lederer. Stability of the spin density wave phases in (TMTSF)2ClO4: quantized nesting effect. Journal de Physique Lettres, 1984, 45 (19), pp.943-952. 10.1051/jphyslet:019840045019094300 . jpa-00232435

\section{HAL Id: jpa-00232435 https://hal.science/jpa-00232435}

Submitted on 1 Jan 1984

HAL is a multi-disciplinary open access archive for the deposit and dissemination of scientific research documents, whether they are published or not. The documents may come from teaching and research institutions in France or abroad, or from public or private research centers.
L'archive ouverte pluridisciplinaire HAL, est destinée au dépôt et à la diffusion de documents scientifiques de niveau recherche, publiés ou non, émanant des établissements d'enseignement et de recherche français ou étrangers, des laboratoires publics ou privés. 
Classification

Physics Abstracts

$71.25-72.15-72.80-75.10$

\title{
Stability of the spin density wave phases in (TMTSF) $)_{2} \mathrm{ClO}_{4}$ : quantized nesting effect
}

\author{
M. Héritier, G. Montambaux and P. Lederer \\ Laboratoire de Physique des Solides (*), Université de Paris-Sud, Centre d'Orsay, \\ 91405 Orsay Cedex, France
}

(Reçu le 21 mai 1984, accepté le 31 juillet 1984)

\begin{abstract}
Résumé. - La stabilité des phases Onde de Densité de Spin dans (TMTSF) ${ }_{2} \mathrm{ClO}_{4}$ s'interprète par de simples arguments d'ajustement de surfaces de Fermi anisotropes : nous montrons que l'ordre des anions, en introduisant de nouvelles réflexions de Bragg perturbe l'ajustement et abaisse la stabilité de l'Onde de Densité de Spin. Nous améliorons l'argument de Gor'kov et Lebed' sur l'effet d'un champ magnétique sur l'ajustement en permettant au vecteur d'onde $\mathbf{Q}(H)$ de s'adapter au champ $H$, si bien que l'ajustement est quantifié. Nous prévoyons que $H$ induit une succession de phases magnétiques, où $\mathbf{Q}(H)$ varie linéairement, séparées par des transitions du premier ordre où $\mathbf{Q}(H)$ est discontinu. A basse température, la tension de Hall est constante à l'intérieur d'une phase, comme dans l'Effet Hall Quantifié, en accord avec les données d'effet Hall.
\end{abstract}

\begin{abstract}
The stability of the SDW phases in (TMTSF) ${ }_{2} \mathrm{ClO}_{4}$ can be interpreted by simple arguments of anisotropic Fermi surfaces nesting : first, we show that anion ordering, by introducing new Bragg's reflexions, perturbs the nesting and lowers the SDW stability. Then, we improve Gor'kov and Lebed's argument on the effect of a magnetic field on nesting by allowing the wave vector $\mathbf{Q}(H)$ to adapt to the field so that nesting is quantized. We predict that $H$ induces a succession of SDW phases with $\mathbf{Q}(H)$ varying linearly, separated by first order transitions with $\mathbf{Q}(H)$ discontinuities. At low $T$, the Hall voltage is constant within in SDW phase, as in the Quantized Hall Effect. This is in agreement with the Hall effect data.
\end{abstract}

\section{Introduction.}

In the anisotropic organic conductor (TMTSF) ${ }_{2} \mathrm{ClO}_{4}$, the non centro-symmetric anions undergo an ordering transition at $24 \mathrm{~K}$ [1]. Depending on the rate of cooling through the ordering temperature, different states can be obtained, with different stabilities of the Spin Density Wave phase [2-4]. In rapidly quenched samples in which the anions are completely disordered, the stable phase is a SDW phase, at ambient pressure, up to $4.2 \mathrm{~K}$ and the normal metallic phase at higher temperature. In relaxed crystals, with well ordered anions, the stable phase at ambient pressure and low temperature is superconducting, with a critical temperature $T_{\mathrm{c}} \simeq 1.2 \mathrm{~K}$ and an upper critical field in the $c^{*}$ direction $H_{\mathrm{c} 2} \simeq 0.8 \mathrm{kG}$ [5]. However, the SDW phase can be restored by applying a large enough magnetic field in the $c^{*}$ direction. At a threshold field which depends on temperature (of the order of $40 \mathrm{kG}$ ), a continuous transition from the normal metallic phase to a

(*) Associé au CNRS. 
SDW semimetallic phase is observed [6-8]. In a field larger than $40 \mathrm{kG}$ and below $0.7 \mathrm{~K}$, steps and plateaux of the Hall resistance have been observed $[8,9]$. They have been interpreted either as a cascade of phase transitions $[8,10]$ or as a quantized Hall effect [8]. Stimulated by new experimental findings [9] at an early stage of their development, we have attempted to understand them in a more detailed way.

In order to explain these effects, one may think of two kinds of theoretical interpretations. Given the strongly anisotropic band structure $\left(t_{a} \gg t_{b} \gg t_{c}\right.$, for the electronic transfer integrals in the three cristallographic axes), there exists a $3 \mathrm{D} \rightarrow 1 \mathrm{D}$ cross-over temperature $T_{\text {co }}$, above which the crystal can be considered as one-dimensional, with properties determined by large amplitude thermal and quantum fluctuations. In particular electron interactions are strongly renormalized by those fluctuations [11-12]. Below $T_{\text {co }}$, transverse coupling between one-dimensional chains decouples the Cooper and zero-sound channels, allowing a three-dimensional long range ordering. Therefore the stability of a SDW long range order depends, in principle, on two different factors : 1) the renormalized electron coupling at the cross-over temperature (the $g_{i}^{*}\left(T_{\mathrm{co}}\right)^{\prime}$ 's in the " $g$-ology » picture); 2) the details of three-dimensional Fermi surfaces nesting, leading to two different interpretations of the effects of anion ordering and of an applied magnetic field. We have explored the first possibility in a recent paper [13]. Investigating the effect of an applied magnetic field on one-dimensional fluctuations in the $g$-ology model, we found, indeed, that the SDW stability domain is enlarged by spin effects, but for fields larger than $T_{\text {co }}$, which seems higher than the experimental threshold fields. Moreover the anisotropy of the effect was not correctly given (experimentally, the field component parallel to $b^{*}$ does not have any effect). The purpose of this Letter is to show that anion ordering and magnetic field effects are closely related and can be coherently interpreted by simple arguments of three-dimensional anisotropic Fermi surfaces. Mutatis mutandis, the same kind of arguments are believed to apply to related compounds, in particular to (TMTSF) ${ }_{2} \mathrm{PF}_{6}$.

\section{Effect of anion ordering.}

In this section, we propose to explain why a Spin Density Wave is stable at ambient pressure and low temperature in the Quenched state, while this is not the case in the Relaxed state.

The essence of our interpretation is to describe ordering of anions by an additional Bragg reflexion in the transverse direction and to neglect the anion potential when they are disordered. We assume that renormalized electron interactions at $T_{\text {co }}$ favour SDW rather than charge density wave ordering. The unstability of the normal metallic phase with respect to SDW formation is discussed in the mean-field approximation assumed to describe correctly the phase diagram in the three-dimensional regime. We do not consider here the competition between superconductivity and magnetism. A procedure consistent with our picture would be to calculate the superconducting critical temperature in the B.C.S. approximation and to compare it to the critical temperature for SDW ordering found here. This is left for future work.

Stoner's criterion gives us the condition for the formation of a SDW phase

$$
1-\lambda \chi^{0}(\mathbf{Q}, T) \leqslant 0
$$

where $\lambda$ is the molecular field and $\chi^{0}(\mathbf{Q}, T)$ is the magnetic susceptibility of non interacting electrons, at wave vector $\mathbf{Q}$ and temperature $T$

$$
\chi^{0}(\mathbf{Q}, T)=\sum_{\mathbf{k}} \frac{f_{T}[\varepsilon(\mathbf{k}+\mathbf{Q})]-f_{T}[\varepsilon(\mathbf{k})]}{\varepsilon(\mathbf{k})-\varepsilon(\mathbf{k}+\mathbf{Q})} .
$$

Here $\varepsilon(\mathbf{k})$ is the dispersion relation of electron with wave vector $\mathbf{k}$ and $f_{T}(\varepsilon)$ is the Fermi distribution at temperature $T$. 
Let us first neglect the effect of anion potential on the electronic structure. Given the anisotropy of the electron transfer integrals, the simplest approximation for $\varepsilon(\mathbf{k})$ is to consider a linear dispersion for wave vectors parallel to the chains and a tight binding one for wave vectors perpendicular to the chains :

$$
\left\{\begin{array}{l}
\varepsilon^{0}(\mathbf{k})= \pm v_{\mathrm{F}}\left(k_{x} \mp k_{\mathrm{F}}\right)+\varepsilon_{\perp}^{0}\left(\mathbf{k}_{\perp}\right) \\
\varepsilon_{\perp}^{0}\left(\mathbf{k}_{\perp}\right)=-2 t_{b} \cos k_{y} b^{*}-2 t_{c} \cos k_{z} c^{*}
\end{array}\right.
$$

Equation (3) describes a perfect Fermi surface nesting, so that the ground state is never metallic [14]. Corrections to equation (3) are basic to understand anion ordering or magnetic field effects since, as will be shown below, neither additional Bragg reflexions, nor magnetic field have first order effects on SDW stability if such corrections are not considered. One must include either correction to linear dispersion along the chains (the band is three-quarter filled) or second harmonics in the Fourier series for $\varepsilon_{\perp}\left(k_{\perp}\right)$

$$
\begin{aligned}
& \varepsilon(\mathbf{k})=\varepsilon^{0}(\mathbf{k})+C\left(k_{x}-k_{\mathrm{F}}\right)^{2} \\
& \varepsilon(\mathbf{k})=\varepsilon^{0}(\mathbf{k})-2 t_{b}^{1} \cos 2 k_{y} b^{*}-2 t_{c}^{1} \cos 2 k_{z} c^{*} .
\end{aligned}
$$

Since $t_{c}^{1} \ll t_{b}^{1}$ dispersion laws $(4 \mathrm{a})$ and (4b) give very similar Fermi surfaces provided

$$
t_{b}^{1}=-\frac{C t_{b}^{2}}{v_{F}^{2}} .
$$

In the following, we consider only case (4b), but the results are expected to apply qualitatively to case $(4 a)$.

Now we consider the anion effects on the electronic structure. In the Quenched state (hereafter noted Q-state), we neglect electron mean free path effects : the anion potential is assumed to have no consequence on SDW stability. In the Relaxed state (R-state), the modulation of wavevector $\left(0, \frac{\pi}{b^{*}}, 0\right)[1]$ doubles the unit cell. The weak potential $\Delta \exp \left(\frac{i \pi}{b^{*}} y\right)\left(\Delta \ll t_{b}\right)$ induces new Bragg reflexions. To first order in perturbation, instead of $(4 b)$, the new dispersion relation is :

$$
\begin{aligned}
\varepsilon^{\mathrm{R}}(\mathbf{k})=\left(\varepsilon^{0}(\mathbf{k})-\varepsilon_{\perp}^{0}(\mathbf{k})\right) \pm\left(\Delta^{2}+\right. & \left.4 t_{b}^{2} \cos ^{2} k_{y} b^{*}\right)^{1 / 2} \\
& -2 t_{c} \cos k_{z} c^{*}-2 t_{b}^{1} \cos 2 k_{y} b^{*}-2 t_{c}^{1} \cos 2 k_{z} c^{*}
\end{aligned}
$$

Our main point is that, with (5), a qualitative difference with the Q-state occurs : the R-state is much more easily metallic. This is found by examination of $\chi^{0}(Q, T)$ for the best nesting wave vector in each case. In the Q-state (dispersion relation Eq. (4b)) the Fermi surface and itself translated by $\mathbf{Q}$ are tangent and have equal curvature radii at the contact point. Thus,

$$
\chi_{\mathrm{Q}}^{0}\left(\mathbf{Q}_{\mathrm{Q}}, T\right) \simeq \frac{1}{8 \pi^{3}} \int \frac{\mathrm{d} \varepsilon \mathrm{d} y \mathrm{~d} z f_{\mathrm{T}}(\varepsilon)}{\varepsilon+\alpha \frac{\left(t_{b}^{1}\right)^{2}}{t_{b}} y^{3}+\beta \frac{\left(t_{c}^{1}\right)^{2}}{t_{c}} z^{3}}
$$

where $\alpha$ and $\beta$ are numerical constants.

In the R-state, equation (5), such a good nesting cannot be achieved because the Fermi surface at the inflexion points has unequal slopes.

$$
\chi_{\mathrm{R}}^{0}\left(\mathbf{Q}_{\mathrm{R}}, T\right) \simeq \frac{1}{8 \pi^{3}} \int \frac{\mathrm{d} \varepsilon \mathrm{d} y \mathrm{~d} z f_{T}(\varepsilon)}{\varepsilon+\alpha^{\prime} \Delta\left(\frac{t_{b}^{1}}{t_{b}}\right)^{2} y^{2}+\beta^{\prime} \frac{\left(t_{c}^{1}\right)^{2}}{t_{c}} z^{3}} .
$$


From equations (6) and (7), one sees that there is no first order effect of anion ordering $(\Delta \neq 0)$ if $t_{b}^{1}=0$ as discussed above. One sees immediately that if $t_{b}^{1} \neq 0$, Q-state exhibits a stronger Kohn anomaly, which implies a higher ordering temperature. To estimate the latter in a rough way, we approximate the energy denominators in (6) and (7) by neglecting the cubic terms. Then, we obtain, in the Q-state, a divergent susceptibility at zero temperature and, therefore, an ordered state for vanishing values of $\lambda$.

$$
T_{\mathrm{SDW}}^{\mathrm{Q}} \sim v_{\mathrm{F}} k_{0} \exp -\frac{v_{\mathrm{F}} k_{\mathrm{F}}}{\lambda}
$$

where $k_{0}$ is a wave vector cut-off in the $k_{y}-k_{z}$ plane.

On the contrary, in the R-state, $\chi_{\mathbf{R}}^{0}(T \rightarrow 0)=A_{0}$ remains finite :

$$
A_{0} \simeq\left(\frac{\eta_{0} t_{b}}{\Delta_{1} t_{b}^{1}}\right)^{1 / 2}\left[\operatorname{Ln} \frac{\omega_{0}}{\eta_{0}}+2\right]
$$

where $\eta_{0}$ is an energy cut-off in the $k_{y}-k_{z}$ plane and $\omega_{0}$ in the $k_{x}$-direction. The existence of an ordered state requires $1-\lambda A_{0}<0$. In such a case, the ordering temperature can be deduced from Rice's work [15] :

$$
T_{\mathrm{SDW}}^{\mathrm{R}} \simeq\left(\frac{\Delta_{1} t_{b}^{1}}{t_{b}}\right)^{1 / 3}\left(A_{0}-\frac{1}{\lambda}\right)^{2 / 3}\left(k_{\mathrm{F}} v_{\mathrm{F}}\right)^{4 / 3}
$$

These rough approximations should not be considered as accurate determinations of the critical temperatures, but rather as qualitative examples to show the effect of the Bragg reflexions due to anion ordering on the Kohn anomaly and therefore on the stability of the Spin Density Wave. We believe that this can explain why the SDW phase is not stable at ambient pressure in the Relaxed state (or at least less stable than the superconducting phase). In the following section, we show that the same corrections to perfect nesting (Eq. (5)) which allows to understand anion ordering effects also allows for a qualitative understanding of the magnetic field effects, provided diamagnetism is taken into account.

\section{Effect of a magnetic field.}

In this section, we study the phase diagram of the Relaxed state in the presence of an applied magnetic field in the $c^{*}$ direction. The basic idea is due to Gor'kov and Lebed' [10]. They have shown, very recently, that orbital effects on the non-interacting susceptibility can improve the nesting and restore the Spin Density Wave. However, they did not choose the best wave vector. For that reason, they were unable to give a completely satisfactory phase diagram. In the following, we show that, by allowing the wave vector of the Spin Density Wave to adapt to the magnetic field, we can predict a phase diagram which accounts more satisfactorily for the experimental data. As pointed out by Gor'kov and Lebed', the argument can be understood qualitatively by examining the semiclassical motion in the direct space of a wave packet at the Fermi surface when a magnetic field is applied in the $c^{*}$ direction. It moves at constant velocity $v_{\mathrm{F}}$ in the $x$-direction, but oscillates in the perpendicular direction. The motion is nearly one-dimensional when the amplitude of the oscillations is smaller than $b^{*}$ i.e. when $2 c t_{b} / e H v_{F} b^{*}<1$, but a Fermi surface warping of amplitude $2 t_{b}^{1}$ is already suppressed when $c t_{b}^{1} / e H v_{\mathrm{F}} b^{*}<1$. Such a qualitative argument applies to dispersion relation (4b) (and therefore to (5) when the anion potential is taken into account). It seems to apply as well in the case (4a). However, in such a case the rigorous calculations of $\chi^{0}(Q, T)$ is less simple. Therefore, in the following, we consider only the case (4b), 
that is to say the dispersion relation (5) in the Relaxed state. We look for the best SDW wave vector in the presence of a magnetic field

$$
\mathbf{Q}(H)=\left(2 k_{\mathrm{F}}+q_{x}, \frac{\pi}{b^{*}}+q_{y}, \frac{\pi}{c^{*}}+q_{z}\right) .
$$

First, we assume that, in general, for most values of the field $\mathbf{Q}(H)$ is different enough from $\mathbf{Q}_{0}=\left(2 k_{\mathrm{F}}, \frac{\pi}{b^{*}}, \frac{\pi}{c^{*}}\right)$ so that one can approximate the dispersion relation (5) by equation (3). Taking into account the or bital effect of the field on the one electron Green's function and therefore on $\chi^{0}(\mathbf{Q}, T)$, it is straightforward to extend the calculations of reference [10] to a general wave vector $\mathbf{Q}(H)$. We find the following Stoner criterion for the stability of the SDW phase :

$\frac{1}{\zeta}-\int_{d}^{\infty} J_{0}\left[\frac{8 c}{e H} \frac{t_{b} \sin q_{y} b^{*} / 2}{v_{\mathrm{F}} b^{*}} \sin \left(\frac{e H b}{2 c} x\right)\right] \cdot J_{0}\left[\frac{8 t_{c} \sin q_{z} c^{*} / 2}{v_{\mathrm{F}}} x\right] \times$

$$
\times \cos \left(q_{x} x\right) \times \frac{2 \pi T \mathrm{~d} x}{v_{\mathrm{F}} \operatorname{sh} \frac{2 \pi T x}{v_{\mathrm{F}}}} \leqslant 0
$$

where $\zeta=\frac{\lambda}{2 \pi v_{\mathrm{F}} b^{*} c^{*}}$ and $d$ is the lower distance cut-off.

The largest value of the integral in (10) is obtained for $q_{z}=0$.

Then, the Stoner criterion can be written :

$$
\operatorname{Ln} \frac{4 t_{b} \sin q_{y} b^{*} / 2}{\pi T_{0}} \lesssim \int_{1}^{\infty} J_{0}\left[\frac{8 c}{e H} \frac{t_{b} \sin q_{z} b^{*} / 2}{v_{\mathrm{F}} b^{*}} \sin y\right] \cdot(\cos 2 v y) \times \frac{\frac{2 \pi T}{v_{\mathrm{F}}} \frac{2 c}{e H b^{*}} \mathrm{~d} y}{\operatorname{sh} \frac{2 \pi T}{v_{\mathrm{F}}} \frac{2 c}{e H b^{*}} y}
$$

where $T_{0}$ is the transition temperature corresponding to the dispersion relation (3) in zero field. In equation (11) we have set

$$
v=\frac{q_{x} c}{e H b^{*}}=\left(q_{x} \frac{2 \pi}{b^{*}}\right) /\left(\frac{e H 2 \pi}{c}\right)=\frac{S\left(q_{x}\right)}{S_{0}}
$$

$v$ is the ratio of the area $S\left(q_{x}\right)$ enclosed between the Fermi surface and this surface translated by $\mathbf{Q}$ to the quantum of momentum space area $S_{0}$ in the presence of a field $H$.

The SDW wave vector is determined by the condition that $\chi^{0}(\mathbf{Q}, T)$ and, therefore, the integral in (11) is maximum. It can be shown that this integral is convergent even in the limit $T \rightarrow 0$ for any value of $v$, except if $v$ is an integer. In that case, setting $v=n$, we average over the oscillations of the Bessel function argument, using the relation :

$$
\frac{1}{\pi} \int_{0}^{\pi} J_{0}[2 z \sin y] \cos 2 n y \mathrm{~d} y=J_{n}^{2}(z) \text {. }
$$

The integral diverges logarithmically at zero temperature. Then, the Stoner criterion defines a critical temperature $T_{n}$ for SDW formation for each integer value of $v$

$$
\log \frac{2 \pi T_{n} 2 c}{e H b^{*} v_{\mathrm{F}}}=\left(\log \frac{\pi T_{0}}{4 t_{b} \sin q_{y} \frac{b^{*}}{2}}\right) /\left(J_{n}\left[\frac{4 c t_{b} \sin q_{y} b^{*} / 2}{e H v_{\mathrm{F}} b^{*}}\right)^{2} .\right.
$$


At a given $n, q_{y}$ is determined by the condition that $T_{n}$ is maximum, i.e. the argument of the Bessel function $z \sim n$.

This corresponds to

$$
\frac{4 t_{b}}{v_{\mathrm{F}}} \sin q_{y} \frac{b^{*}}{2}=q_{x}
$$

when

$$
q_{x} \leqslant \frac{4 t_{b}}{v_{\mathrm{F}}} \quad \text { or } \quad \frac{e H}{c} \leqslant \frac{4 t_{b}}{v_{\mathrm{F}} b^{*}} \frac{1}{n}
$$

and $\sin q_{y} \frac{b^{*}}{2}=1$ at higher fields.

The physical meaning is clear : the Spin Density Wave of wave vector $\mathbf{Q}$ leaves a pocket of unpaired charge carriers. The section in the $\left(k_{x}, k_{y}\right)$ plane of this pocket has an area $S\left(q_{x}\right)$ which is quantized in the presence of a magnetic field parallel to $c^{*}$. Condition (12) tells us that the pocket involves an integer number of completely filled Landau levels. Condition (14) tells us that the best nesting is obtained when the Fermi surface and itself translated by $\mathbf{Q}$ are tangent. When the field increases, the degeneracy of the Landau levels increases linearly, and from (12), so does $S\left(q_{x}\right)$. At the same time, $Q_{x}$ and $Q_{y}$ vary so that the surfaces remain tangent while the quantized orbits inflate : quantization of nesting is preserved. In this field range, the argument of the Bessel function $z \simeq n$. Eventually, when $Q_{x}>2 k_{\mathrm{F}}+4 \frac{t_{b}}{v_{\mathrm{F}}}\left(\right.$ and $\left.Q_{y}=0\left(\bmod \frac{2 \pi}{b^{*}}\right)\right)$, the surfaces become separated and $z$ decreases.

However, to derive equation (13) we have assumed that the dispersion law could be approximated by equation (3). This becomes invalid at too small values of $q_{x}$ and $q_{y}$, i.e. for a given value of $n$, at small field. The validity condition of (13) is

$$
q_{y} \frac{b^{*}}{2} \gtrsim \frac{t_{b}^{1}}{t_{b}} \quad \text { or } \quad v_{\mathrm{F}} q_{x} \gtrsim 4 t_{b}
$$

that is to say $\frac{e H}{c} \gtrsim \frac{4 t_{b}^{1}}{v_{\mathrm{F}} b^{*}} \frac{1}{n}$.

In the opposite limit $\frac{e H}{c} \ll \frac{4 t_{b}^{1}}{v_{\mathrm{F}} b^{*}} \times \frac{1}{n}$, we can assume that $\varkappa^{0}(\mathbf{Q}, T)$ is essentially determined by the terms in $t_{b}^{1}$ in the dispersion relation because the terms in $t_{b}$ cancel out when $\mathbf{Q} \rightarrow \mathbf{Q}_{0}$. In that case, one must write, instead of equation (13) :

$$
\left\{\begin{array}{l}
\log \frac{2 \pi T_{n} c}{e H b^{*} v_{\mathrm{F}}}=\left(\log \frac{\pi T_{0}}{4 t_{b}^{1}}\right) /\left(J_{n}\left[\frac{2 c t_{b}^{1}}{e H v_{\mathrm{F}} b^{*}}\right]\right)^{2} \\
q_{y} \simeq 0 .
\end{array}\right.
$$

Here $n=q_{x} \frac{c}{e H 2 b^{*}}$.

Two important differences with equation (13) must be pointed out.

i) The ratio $S\left(q_{x}\right) / S_{0}=2 n$ must be, now, an even number. Indeed, because the $t_{b}{ }^{1}$ term has a period $\pi / b^{*}$ (instead of the period $2 \pi / b^{*}$ of the $t_{b}$ term), the unpaired charge carrier pocket is split in two equal parts by the $t_{h}^{1}$ term when $q_{x} \lesssim t_{h}^{1} / v_{\mathrm{F}}$. Solutions involving an even number of Landau levels at high field remain stable at low field when the pocket is split in two half-pockets, 
each of them involving the same number of Landau levels. However, this is obviously impossible for solutions involving an odd number of Landau levels, which therefore become unstable for

$$
\frac{e H}{c} \lesssim \frac{4 t_{b}^{1}}{v_{\mathrm{F}} b^{*}} \frac{1}{n} .
$$

ii) A second consequence of equation (15) is that the argument $z$ of the Bessel function is now an increasing function of $1 / H$.

Figure 1 shows schematically the variations of $J_{2 p}^{2}(z), J_{2 p-1}^{2}(z)$ and $J_{2 p-2}^{2}(z)$. The stable phase, characterized by $\mathbf{Q}$, i.e. by $v$ corresponds to the higher value of the Bessel function. Therefore, the intersections shown in figure 1 signal phase transitions. In our approximation, they are obtained for

$$
\frac{e H}{c} \simeq \frac{4 t_{b}^{1}}{v_{\mathrm{F}} b^{*}} \frac{1}{2 p+1}
$$

and

$$
\frac{e H}{c} \simeq \frac{4 t_{b}^{1}}{v_{\mathrm{F}} b^{*}} \frac{1}{2 p}+z_{2 p}
$$

where $z_{2 p}$ is defined by

$$
J_{2 p}\left(z_{2 p}\right)=\operatorname{Max}\left(J_{2 p+1}(z)\right) \text {. }
$$

However, these transitions occur in a field range where $\Delta, t_{b}^{1}$ and $t_{b}$ are important in the determination of $\chi^{0}(\mathbf{Q}, T)$, i.e. when our calculation becomes rather poor. Therefore, the accurate values of the transition fields should not be taken too seriously.

We obtain the phase diagram shown qualitatively in figure 2. At a given field, a second-order transition from a semi-metallic SDW phase to a metallic phase at a temperature given by equation (13) is predicted, as observed in specific heat experiments [6]. At a given temperature, as the field is increased, we obtain, first, a second-order transition from metal to semi-metallic SDW phase, then a cascade of first-order transition between different SDW phases. Although the theory is strictly valid for the metal-SDW transition line only, the existence of this cascade is inferred from a straightforward Landau-Ginzburg expansion, following which, within each phase, the wave vector varies continuously so that the number of unpaired charge carriers is proportional to the field, but is discontinuous at the transition. The description of the low $T$

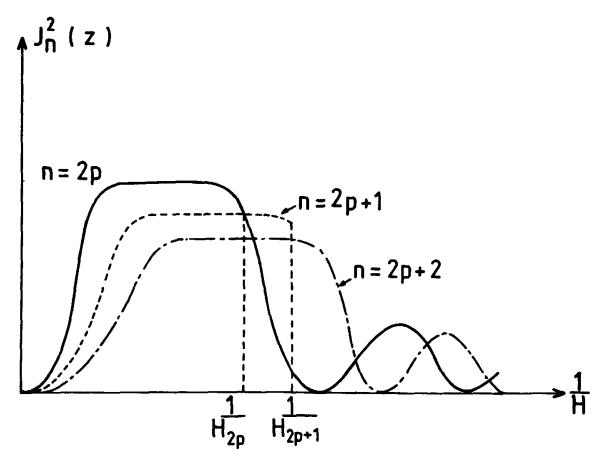

Fig. 1. - Variations of the Bessel functions $J_{n}^{2}(z)$ determining the critical temperatures $T_{n}$, versus $1 / H$. The phase corresponding to $n=2 p+1$ is stable for $H_{2 p+1}<H<H_{2 p}$. 


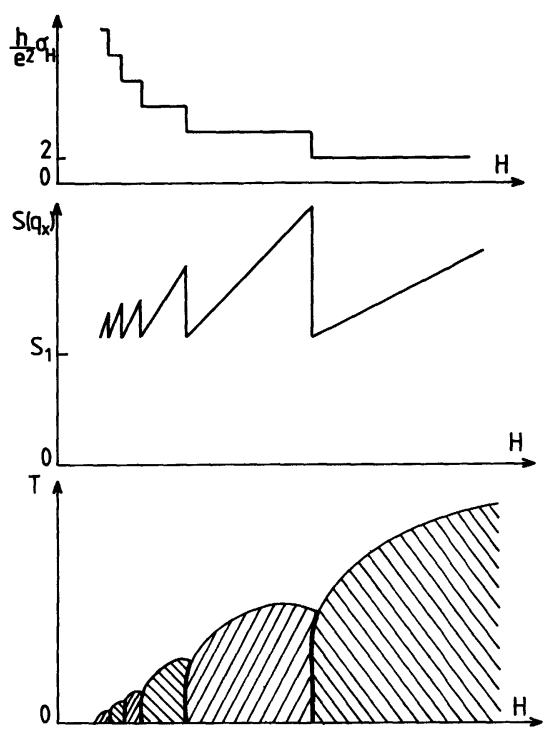

Fig. 2. - Phase diagram inferred from our calculations. Within each shaded area, a different SDW is stable. The heavy línes represent 1 st order transitions between SDW phases. The thin line represents 2 nd order transition from SDW to metallic phase. The low temperature part of the phase diagram is plausible, although not derived quantitatively from the theory. Within each SDW phase, the area of unpaired charge carrier Fermi surface, $S\left(q_{x}\right)$ varies linearly with the field (the minimum possible value $S_{1}=8 \pi t_{b}^{1} / v_{\mathrm{F}} b^{*}$ is never reached) and the Hall conductance $\sigma_{\mathrm{H}}$ is constant (here, a possible valley degeneracy has not been considered).

part of the phase diagram requires a theory of the ordered phases. Taking into account the $t_{c}$ term in the dispersion relation limits the number of different SDW phases which can be obtained in practice. The maximum value $n_{0}$ of $n$ is defined by :

$$
\left[J_{n_{0}}\left(n_{0}\right)\right]^{2} \simeq \frac{\log \frac{\pi T_{0}}{4 t_{b}^{1}}}{\log \frac{t_{c}^{1}}{t_{b}^{1}}} .
$$

This corresponds to a threshold field for SDW formation at $T=0$

$$
H_{0} \sim \frac{4 t_{b}^{1} c}{e v_{\mathrm{F}} b^{*}} \times \frac{1}{n_{0}} .
$$

Equation (12) is a quantized relationship valid along the metal SDW transition line. Although the theory of the low $T$ Hall conductance requires that of the ordered phase, this quantification suggests the explanation for the observed Hall conductance plateaux, as shown in figure 2 : within a phase, it is quantized according to the values $\sigma_{\mathbf{H}}=\left(e^{2} / h\right) g n$, where $g$ is a degeneracy factor (including the spin degeneracy). The physical reason is that the SDW wave vector can adapt itself to the field variation so that the quantization of the Fermi surface nesting is preserved. The electron-hole paired states act as a reservoir to fill in completely the Landau levels. Energetically, the diamagnetic energy is minimum when the Landau levels are filled. This is realized at the expense of a pairing energy per electron $\sim k_{\mathrm{B}} T_{n}$, which, from (13) is always smaller than the 
diamagnetic energy decrease. However, this mechanism lowers the electron-hole condensation energy and therefore the ordering temperature. When the wave vector departs too much from the best zero-field nesting wave vector, one jumps to a different value of $n$. Therefore, when the field is varied, one expects the wave vector to oscillate around the best nesting wave vector (with discontinuities corresponding to the change of integer value $n$ ).

The interpretations of the recent Hall effect data in (TMTSF) ${ }_{2} \mathrm{ClO}_{4}$ put forward in the literature are either a cascade of phase transitions or a quantized Hall effect. According to our picture the phenomenon is a synthesis of these two mechanisms. A cascade of first order transitions indeed occurs, which are necessary to preserve the quantization of the unpaired electron Fermi surface. Because of the continuous (within the plateaux) and discontinuous (between them) variations of $\mathbf{Q}(H)$, the SDW pairing realizes a quantization of the Hall conductance. This Quantized Nesting Effect implies some differences with the standard Quantized Hall Effect. Associated with the first order transitions, hysteresis (of weak amplitude because the free energy differences are rather weak) are expected (and indeed observed). We also expect oscillations of the longitudinal conductivity of much smaller amplitude because the energy difference between the Fermi level and the highest filled Landau level oscillates with the field but without ever vanishing. Another qualitative difference with the Quantized Hall Effect is the positions of the steps which are only approximately periodic in $\frac{1}{H}$ and depend on the details of the real system (electron localization due to disorder, interactions, size effects, etc...). However the quantization of the Hall conductance results immediately, as in the standard Quantized Hall Effect, from gauge invariance. Observing that in a change of gauge, the electron Green's function $G_{++}$is multiplied by a phase factor $\exp \left(i \frac{e}{c} A\left(x-x^{\prime}\right)\right)$, one can reproduce exactly Laughlin's arguments [16] and show that the quantization of the Hall plateaux of the ideal system are universal quantities and are not affected by the detailed properties of the real system.

Recent papers have discussed the Quantized Hall Effect in a two-dimensional periodic potential [17]. The model we use here is different from the ones used in reference [17] in the sense that :

a) periodicity is taken into account in the transverse direction only,

b) electron-electron interaction is present and taken into account in the molecular field approximation,

c) as a result, the intensity of the relevant field in our case is of order $t_{b}^{1}$, orders of magnitude smaller than the ones considered in [17]. Nevertheless, our model also exhibits the possibility of a succession of positive and negative quantized plateaux; the reason is that in the Relaxed state, nesting may produce either electron or hole pocket [18]. This behaviour is not related to a fractional filling of the Landau levels.

In conclusion, we believe that we have given plausible explanations of almost all experimental data on the stability of SDW in (TMTSF) ${ }_{2} \mathrm{ClO}_{4}$. We have shown that anion ordering effects and magnetic field effects are closely connected. In our view, they cannot be discussed separately : anion ordering drives the system away from 1-D like nesting, while the magnetic field drives it closer. A quantitative check of our proposal for the phase diagram under magnetic field requires a detailed theory of the ordered phases.

\section{Acknowledgments.}

We warmly thank P. Garoche, D. Jérome and M. Ribault for numerous helpful discussions during the completion of this work and for communicating their data prior to publication. A discussion with $\mathrm{G}$. Toulouse is also gratefully acknowledged. 


\section{References}

[1] Pouget, J. P., Shirane, G., Bechgaard, K. and Fabre, J. M., Phys. Rev. B 27 (1983) 5203.

[2] Takahashi, T., Jérome, D. and Bechgaard, K., J. Physique Lett. 43 (1982) L-565.

[3] Tomic, S., Jérome, D., Monod, P. and Bechgaard, K., J. Physique Lett. 43 (1982) L-839.

[4] Garoche, P., Brusetti, R. and BechgaArd, K., Phys. Rev. Lett. 49 (1982) 1346.

[5] Bechgaard, K., Carneiro, K., Olsen, M., Rasmussen, F. and Jacobsen, C. S., Phys. Rev. Lett. 46 (1981) 852.

[6] Garoche, P., Brusetti, R., Jérome, D. and Bechgaard, K., J. Physique Lett. 43 (1982) L-147 ; Brusetti, R., Garoche, P. and BeChgaARd, K., J. Phys. C 16 (1983) 3525.

[7] Takahashi, T., Jérome, D., BechgaArd, K., J. Physique Colloq. 44 (1983) C3-805.

[8] Ribault, M., Jérome, D., Tuchendler, J., Weyl, C. and BechgaArd, K., J. Physique Létt. 44 (1983) L-953.

Kwak, J. F., Schirber, J. E., Greene, R. L. and Engler, E. M., Phys. Rev. Lett. 46 (1981) 1296.

Chaikin, P. M., Choi, M. Y., Kwak, J. F., Brooks, J. S., Martin, K. P., Naughton, M. J., Engler, E. M. and Greene, R. L., Phys. Rev. Lett. 51 (1983) 2333.

[9] Ribault, M., Cooper, J., Jérome, D., Mailly, D., Moradpour, A. and Bechgaard, K., J. Physique Lett. 45 (1984) L-935 (this issue).

[10] Gor'kov, L. P. and Lebed', A. G., J. Physique Lett. 45 (1984) L-433.

[11] JÉrome, D. and Schulz, H. J., Adv. Phys. 31 (1982) 299.

[12] Solyom, J., Adv. Phys. 28 (1979) 201.

[13] Montambaux, G., Héritier, M. and Lederer, P., J. Physique Lett. 45 (1984) L-533.

[14] Horovitz, B., Gutfreund, H. and Weger, M., Phys. Rev. B 12 (1975) 3174.

See also Yamaj, K., Proceedings of the 6th Taniguchi Symposium (Kashikojima, Nov. 1983).

[15] Rice, T. M., Phys. Rev. B 2 (1970) 3619.

[16] Laughlin, R. B., Phys. Rev. B 23 (1981) 5632.

[17] Thouless, D. J., Kohmoto, M., Nightingale, M. P. and Den Nius, M., Phys. Rev. Lett. 49 (1982) 405.

[18] Ribault, M. et al., to be published. 